\title{
Initial Fluid Resuscitation Following Adjusted Body Weight Dosing in Sepsis and Septic Shock
}

\author{
Oana Antal1,2*, Elena Ștefănescu|1,2, Monica Mleșnițe ${ }^{1,2}$, Andrei Mihai Bălan ${ }^{1}$, Natalia \\ Hagău1,2 \\ 1 Department of Anaesthesia and Intensive Care, "Iuliu Hațieganu" University of Medicine and Pharmacy, \\ Cluj-Napoca, Romania \\ 2 Department of Anaesthesia and Intensive Care, Emergency Clinical County Hospital, Cluj-Napoca, Romania
}

\begin{abstract}
Introduction: Fluid administration is considered a fundamental part of early sepsis treatment. Despite abundant research, fundamental questions about the amount of fluids to be given remain unanswered. Recently, the idea of adjusting the fluid load to the ideal body weight emerged, as obesity rates are increasing, and fluid overload was proven to increase mortality.

Aim of the study: The study aimed to determine whether advanced haemodynamic monitoring supports the adjustment of the initial fluid load to the ideal body weight (IBW).

Methods: Seventy-one patients with sepsis and septic shock were enrolled in the study. The initial fluid resuscitation was performed using local protocols. The haemodynamic status was assessed after the initial fluid load by transpulmonary thermos-dilution technique and the renal outcome recorded at twenty-four hours.

Results: $68.6 \%$ of the patients included in the study had weight disorders ranging from $\mathrm{BMI}+20 \%$ to morbid obesity. Before IBW adjustment, only 49.3\% received the $30 \mathrm{ml} / \mathrm{kg}$ fluid load recommended by Surviving Sepsis Campaign Guidelines (2016) (SSC). After IBW adjustment, 70.4\% received the recommended fluid dose. The difference in fluid $\mathrm{load} / \mathrm{kg}$ before and after the bodyweight adjustment was statistically significant $(p<0.01)$. After the initial fluid load, the majority of the macro haemodynamic parameters were in the targeted range. There was no statistically significant difference between the urinary output outcome at 24 hours or the 28 days mortality rates between the patients resuscitated by the SSC and those who received less fluid.

Conclusions: Advanced haemodynamic monitoring was in favour of adjusting the initial fluid load to the IBW. There were no statistically significant differences either in the urinary output outcome at twenty-four hours, or in the twenty-eight-day mortality rates between the patients who received the $30 \mathrm{ml} / \mathrm{kg} \mathrm{IBW}$ and those who received less than $30 \mathrm{ml} / \mathrm{kg}$ IBW.
\end{abstract}

Keywords: ideal body weight fluid adjustment, advanced haemodynamic monitoring

Received: 1 June 2019 / Accepted: 28 October 2019

\section{INTRODUCTION}

Sepsis continues to be a leading cause of mortality and morbidity among critically ill patients. Even though fatality rates have improved over the last decades, mortality remains high [1]. Fluid administration is considered a fundamental part of early sepsis treatment [2]. In recent years there have been several important clinical trials carried out on this subject. Despite this abundant research, fundamental questions about the amount of fluids to be given remain unanswered [3].
A few years ago, a new approach regarding the management of these conditions emerged: personalized medicine, which is considered to be the future of intensive care medicine $[4,5]$. This concept includes personalized haemodynamic management of critically ill patients. Therefore, measuring haemodynamic variables, such as cardiac output, stroke volume, extravascular lung water or global end-diastolic volume, and guiding the fluid therapy accordingly, should be considered as part of the treatment of septic patients. Moreover, adjusting the macro-haemodynamic parameters is the 
first step in achieving "haemodynamic coherence", an important pillar in the management of the critically ill patients [4].

The study aimed to determine to what extent the advanced haemodynamic monitoring influences the management of fluid resuscitation.

\section{- PATIENTS AND METHODS}

This was a prospective observational study carried out between January 2016 and July 2017, in a university hospital (The Emergency County Hospital Cluj, ClujNapoca, Romania).

The Ethics Committee of the University of Medicine and Pharmacy of Cluj-Napoca approved the study protocol (no 119/6.03.2015). Before data acquisition, informed consent was obtained from each patient or their next of kin, if the patient was unable to give consent.

\section{Study patients}

Seventy-one patients with severe sepsis and septic shock, initially defined according to the Surviving Sepsis Campaign Definitions [6] and subsequently redefined according to the new Sepsis-3 definitions in sepsis and septic shock [7], were included in the study.

Patients were recruited either in the emergency department (ED) or the hospital ward.

Patients were excluded if they were $>80$ years old, had severe cardiac disease as defined by the New York Heart Association (NYHA) IV stage, significant aortic valvular disease, stage IV chronic obstructive pulmonary disease, Child-Pugh score $\mathrm{C}$, known vascular disease, abnormal Allen test, severe anaemia, those with religious believes which impeded blood transfusions, severe hypothermia, patients requiring "prone positioning", end-stage neoplastic diseases, soft tissue infection at the site of catheter insertion, chronic sepsis or patient refusal.

These complex exclusion criteria were used in order to avoid all factors that could bias the haemodynamic of the patients [8-11].

Both spontaneous breathing and mechanically ventilated patients were included in the study.

\section{Data Collection}

The time of presentation was taken to be the time of the first charted recording, which suggested sepsis [12].
Time zero was taken to be the time when a patient was admitted to the ICU.

All patients were haemodynamically resuscitated from the time of presentation to the third hour after study inclusion according to local protocols, without using any advanced haemodynamic monitoring. Both crystalloid and colloid solutions were given and administered both through peripheral and central lines. From the third hour to the twenty-fourth hour after study inclusion, all patients continued to be resuscitated using minimally invasive haemodynamic monitoring parameters: stroke volume variation (SVV) at passive leg raising (PLR), cardiac index (CI), systemic vascular resistance index (SVRI), global end-diastolic index (GEDI), intrathoracic blood index (ITBI), extravascular lung index (ELWI), global ejection fraction (GEF) obtained on transpulmonary thermodilution analysis (EV1000 clinical platform, Edwards Lifesciences@ Irvine, CA, USA). Calibrations were performed at 3, 6 and 24 hours after study inclusion, and at any time the vasoactive infusion was adjusted.

In order to use this haemodynamic monitoring system, a central venous line was placed on the jugular or subclavian vein and an arterial catheter on the femoral artery. The vessel to be cannulated was chosen after performing an echography of the arteries and veins, always aiming for normal blood flow.

Alongside the advanced haemodynamic monitoring, we also used static haemodynamic parameters such as blood pressure (BP), central venous pressure (CVP) and also clinical features such as urinary output (UO) and capillary refill time (CRT).

The study protocol is summarized in figure 1 .

The vasopressor dependency index (VDI), a surrogate for the haemodynamic impairment, was used to express the relationship between the vasopressor infusion dose and mean arterial blood pressure (MAP). VDI is calculated using the formula: ((Dobutamine dose 1 ) $+\left(\right.$ Dopamine dose $\left.e^{\star} 1\right)+\left(\right.$ Norepinephrine dose $\left.\mathrm{d}^{\star} 100\right)+$ $\left(\right.$ Vasopressin $\left.^{\star} 100\right)+\left(\right.$ Epinephrine $\left.\left.^{\star} 100\right)\right) /$ MAP, where Epinephrine, Norepinephrine, Dobutamine and Dopamine are expressed as $\mu \mathrm{g} / \mathrm{kg} / \mathrm{min}$ and Vasopressin as units/min.

SOFA, cardiovascular SOFA and APACHE II scores were used to classify the illness severity $[13,14]$.

The KDIGO and AKIN urinary output criteria were used to define sepsis-related AKI at 24 hours after study inclusion $[15,16]$. 


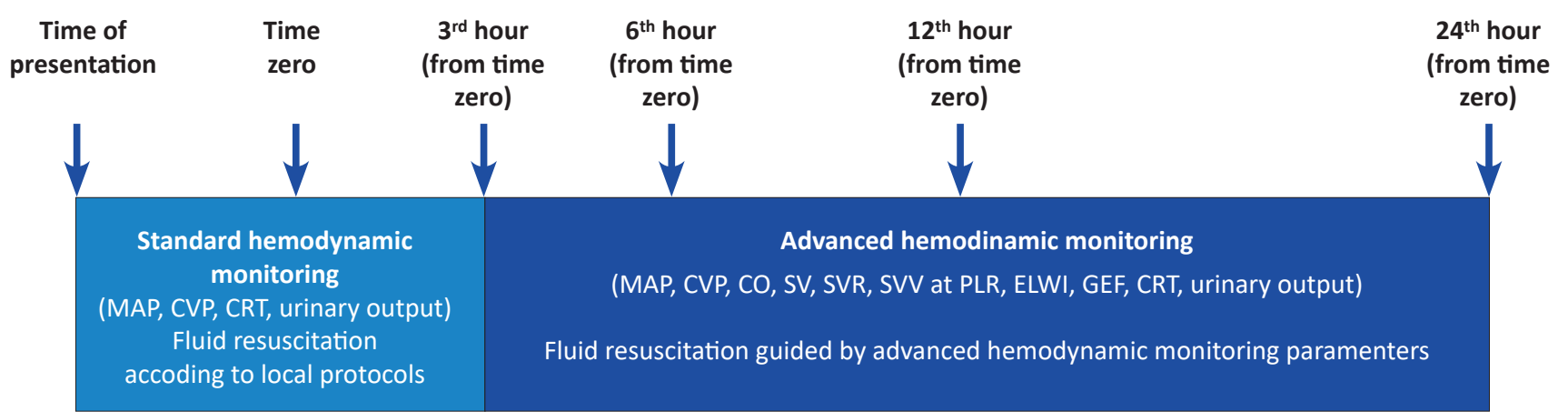

Fig. 1. Study protocol. Time of presentation: time of the first chart recording of sepsis; Time zero: time of study inclusion (ICU); MAP: mean arterial blood pressure; CVP: central venous pressure; CO: cardiac output; SV: stroke volume; SVR: systemic vascular resistance; SVV at PLR: stroke volume variation at passive leg rising; ELWI: extravascular lung water index; GEF: global ejection fraction; CRT: capillary refill time

\section{Statistical analysis}

Statistical analysis was performed using IBM SPSS Statistics version 23.0, MedCalc statistical software version 17.9, and Microsoft Excel 2013.

Continuous variables were expressed as mean (SD), while categorical variables were expressed as numbers or percentages. For descriptive statistics, we used tables and graphs. To compare means, the Wilcoxon Signed Rank Test and Mann-Whitney U test were used for data that did not follow a normal distribution and independent samples t-test for the data that was normally distributed.

The level of significance was set at $\alpha=0.05$.

For comparing proportions, the two-proportion ZTest was used.

\section{RESULTS}

From January $1^{\text {st }}, 2016$ to July $31^{\text {st }}, 2017$, seventy-one patients with sepsis and septic shock were enrolled into the study (Figure 2).

All the 71 patients were included in the statistical analysis. Their demographic and physiologic characteristics are shown in table 1.

The Mean Arterial Blood Pressure (MAP) at the time of presentation was $64.61 \mathrm{~mm} \mathrm{Hg} \pm 15.04 \mathrm{~mm} \mathrm{Hg}$ because $46.4 \%$ of patients were already on vasoactive support.

When analysed, the total fluid load received by the patients in the time interval from the time of presentation to the 3rd hour of fluid resuscitation (initial fluid

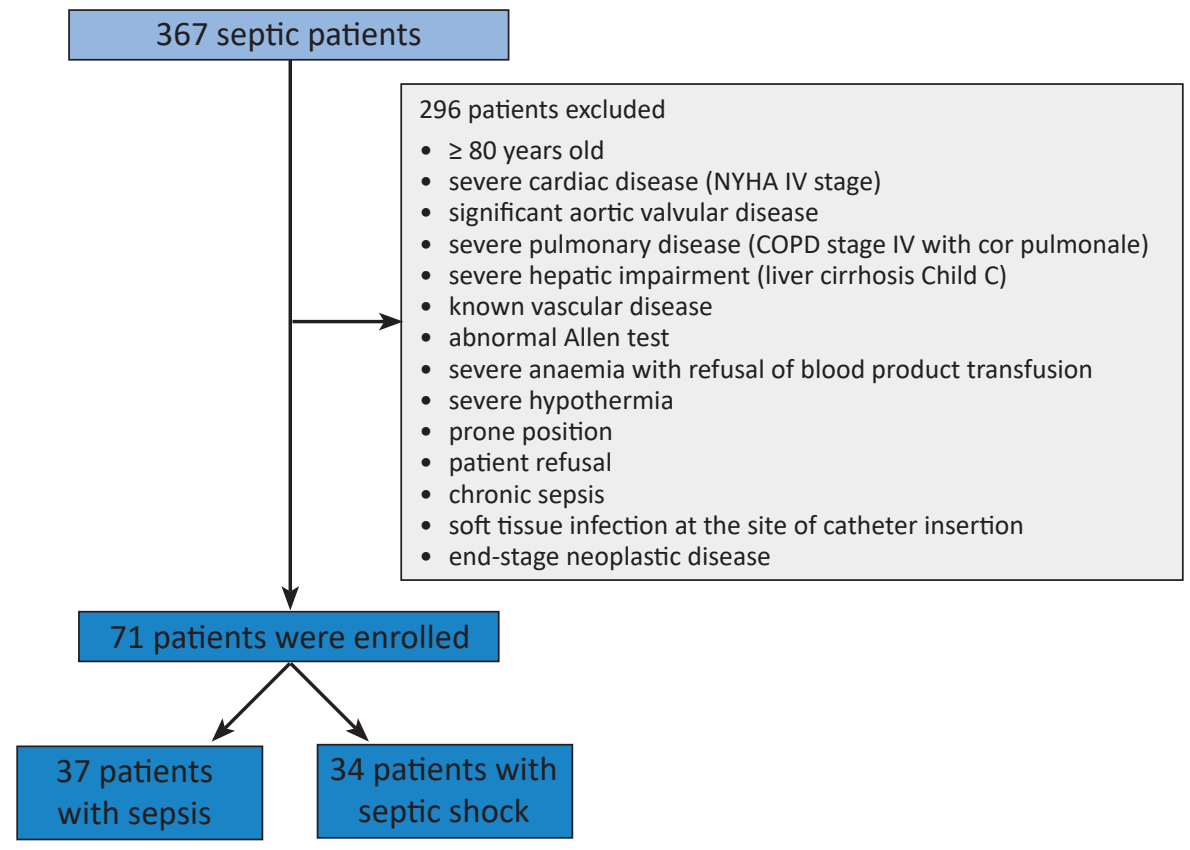

Fig. 2. Flow chart of patient inclusion and exclusion criteria 
resuscitation), it was observed that only $49.30 \%$ of the patients, received $30 \mathrm{ml} / \mathrm{kg}$ body weight as recommended by the SSC 2016 guidelines.

Only $32.4 \%$ were with normal body mass index (BMI), $42.3 \%$ had a weight corresponding to $\mathrm{BMI}+20 \%, 9.9 \%$ had $\mathrm{BMI}+40 \%$ and $15.4 \%$ were with obesity class II and III. After performing the ideal body weight (IBW) according to the Lemmens formula [17], and adjusting the total volume of fluids received in the same time interval to the IBW, we obtained different results. The comparison of the fluid load before and after IBW adjustment is shown in figure 3 .

The percentage of patients receiving $\geq 30 \mathrm{ml} / \mathrm{kg}$ fluid in initial fluid resuscitation increased from $49.40 \%$ before IBW adjustment to $70.4 \%$ afterwards. There was a statistically significant difference between the amount

\section{Table 1. The demographic and physiologic characteristics} of the patients enrolled

\begin{tabular}{lc} 
Diagnosis & $\mathbf{N}(\%)$ \\
\hline Sepsis & $37(52.1 \%)$ \\
Septic shock & $34(47.9 \%)$ \\
Type of sepsis & $\mathrm{N}(\%)$ \\
Medical & $26(36.6 \%)$ \\
Surgical & $45(63.4 \%)$ \\
Sex & $\mathrm{N}(\%)$ \\
Female & $21(29.6 \%)$ \\
Male & $50(70.4 \%)$ \\
Age Mean (SD) & $62.68(14.78)$ \\
Weight kg Mean (SD) & $82.58(20.03)$ \\
Body Surface Area Mean (SD) & $1.92(0.24)$ \\
Ventilation & $\mathrm{N}(\%)$ \\
Mechanically ventilated & $49(69 \%)$ \\
Spontaneous ventilation & $22(31 \%)$ \\
PEEP for Mechanically ventilated at study & $5.69(1.1)$ \\
incluion (time 0) Mean (SD) & \\
SOFA Score at study inclusion (time 0) & $9.58(3.27)$ \\
Mean (SD) & \\
Cardiovascular SOFA at study incluion & $2.85(1.4)$ \\
(time 0) Mean (SD) & \\
APACHE II Score at study incluion (time 0) & $21.94(8.64)$ \\
Mean (SD) & $105.01(20.64)$ \\
Heart Rate at study incluion (time 0) & \\
Mean (SD) beats/min & $64.6(15.0)$ \\
Mean arterial blood pressure (MAP) at & $22.74(20.25)$ \\
presentation Mean (SD) mm Hg & Lactate at study inclusion (time zero) \\
mmol/l & \\
\hline
\end{tabular}

of fluid/kg received by the patients before and after performing the IBW adjustment $(\mathrm{p}<0.01)$.

By the third hour after study inclusion, the majority of the macro haemodynamic parameters were in the targeted range (Table 2).

When the group of patients who received $\geq 30 \mathrm{ml} /$ $\mathrm{kg}$ IBW fluid load (Group 1) and the group of patients which received less than $30 \mathrm{ml} / \mathrm{kg}$ IBW (Group 2) were compared, there were no statistically significant differences in the SOFA, cardio-vascular SOFA, renal SOFA, pulmonary SOFA and APACHE II scores at time of presentation or at 24 hours after study admission. Furthermore, there were no statistically significant differences between the proportions of patients still anuric/ oliguric at twenty-four hours after study inclusion between the two groups. The mortality rates at twentyeight days among the two groups were not significantly different.

The all-cause mortality for all patients included in the study was $30.9 \%$.

\section{DISCUSSION}

The important finding was that an adjustment of the fluid load to the IBW might be necessary in order to avoid fluid overload.

A large percentage of the patients included in the study were either over weighted or obese, and there are no specific guidelines regarding fluid resuscitation in obese septic patients. A recent report showed that the patients receiving the least amount of fluids/ $\mathrm{kg}$ body weight were the obese ones; it was also proposed that a body weight adjustment be made in the obese septic population when calculating the amount of fluid/kg to be administered in the initial fluid resuscitation [18]. After bodyweight adjustment was made for the patients included in our study, the results showed that $70.4 \%$ of the patients received the $30 \mathrm{ml} / \mathrm{kg}$ fluid load recommended by the SSC (2016), compared to only $49.3 \%$ before the IBW adjustment.

If all the targeted macro haemodynamic parameters are shown to be within normal ranges after the initial resuscitation and the tissue perfusion was improved, it can be concluded that minimally invasive haemodynamic monitoring sustains the idea of bodyweight adjustments for the overweighted and obese septic patients. 


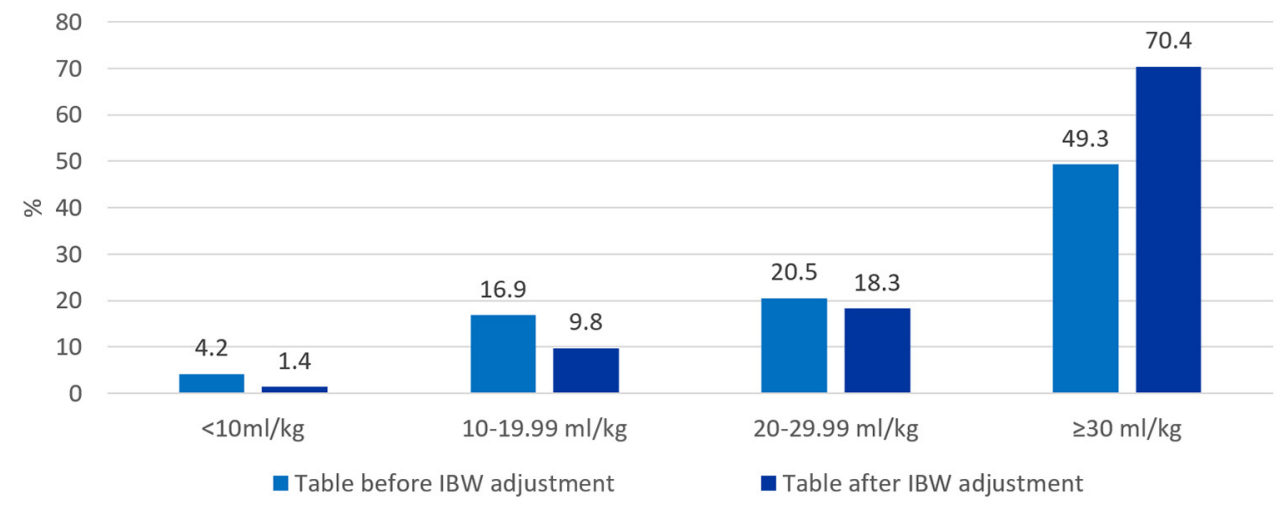

$\mathrm{ml} / \mathrm{kg}$ total fluid load from time of presentation to the 3rd hour after study inclusion (initial fluid resuscitation), before and after performing an ideal body weight adjustment

Fig. 3. Initial fluid load before and after IBW adjustment

The difference in fluid load $/ \mathrm{kg}$ before and after body weight adjustment was statistically significant $(\mathrm{p}<0.01)$. The current findings corroborate previous publications which assert that a restrictive and adjusted fluid therapy in obese patients may have survival benefits $[19,20]$.

When Group 1 was compared with Group 2 no statistically significant differences were found in the SOFA, cardio-vascular SOFA, renal SOFA, pulmonary SOFA and APACHE II scores, either at time of presentation or twenty-four hours after study admission. Patients in the two groups had similar characteristics at these time frames, making the groups suitable for comparison.

Furthermore, there were no statistically significant differences regarding the urinary output outcome at twenty-four hours between the two groups. This can be interpreted as the initial fluid load not being an aggravator factor for the onset or persistence of sepsisinduced AKI. This finding is in concordance with the restrictive fluid therapy approach for the resuscitation of the septic patients $[21,22]$.

Table 2. The haemodynamic parameters of the patients included in the study

\begin{tabular}{|c|c|c|c|c|c|}
\hline $\mathbf{N}=71$ patients & $\begin{array}{c}\text { Time of } \\
\text { presentation }\end{array}$ & $\begin{array}{c}\text { Study inclusion } \\
\text { (time 0) }\end{array}$ & $\underset{\text { value* }}{\mathbf{p}}$ & $3^{\text {rd }}$ hour & $\underset{\text { value }}{\mathbf{p}}$ \\
\hline Heart rate $b / \min (S D)$ & $\#$ & $103.92(18.67)$ & - & $98.7(18.8)$ & 0.10 \\
\hline Systolic blood pressure mm Hg (SD) & $98(22.5)$ & $118.0(21.07)$ & $<0.0001$ & $122.7(18.1)$ & 0.15 \\
\hline Diastolic blood pressure mm Hg (SD) & $49.5(13.6)$ & $56.64(13.13)$ & 0.0003 & $55.5(11.8)$ & 0.60 \\
\hline Mean arterial blood pressure mm Hg (SD) & $64.6(15)$ & $75.22(13.69)$ & $<0.0001$ & $74.8(11.6)$ & 0.86 \\
\hline Central venous pressure $\mathrm{mm} \mathrm{Hg} \mathrm{(SD)}$ & $\#$ & $7.34(4.64)$ & - & $8.5(4.7)$ & 0.13 \\
\hline $\mathrm{Cl}$ l/min Mean (SD) & $\#$ & $\#$ & - & $3.5(0.7)$ & - \\
\hline SVI Mean (SD) ml/m2/beat ) & $\#$ & $\#$ & - & $35.78(10.7)$ & - \\
\hline SVRI Mean (SD) dynes-sec/cm-5/m2 & \# & $\#$ & - & $1691.6(630.06)$ & - \\
\hline GEDI Mean (SD) $\mathrm{ml} / \mathrm{kg}$ & $\#$ & $\#$ & - & $647.5(166.7)$ & - \\
\hline ITBI Mean (SD) ml/m2 & $\#$ & $\#$ & - & $829.7(233.1)$ & - \\
\hline ELWI Mean (SD) Mean (SD) $\mathrm{ml} / \mathrm{kg}$ & $\#$ & $\#$ & - & $8.75(3.76)$ & - \\
\hline Norepinephrine $\mathrm{mcg} / \mathrm{kg} / \mathrm{min}$ & $0.07(0.1)$ & $0.12(0.15)$ & 0.01 & $0.11(0.14)$ & 0.49 \\
\hline VDI & $0.11(0.1)$ & $0.12(0.15)$ & 0.03 & $0.16(0.21)$ & 0.54 \\
\hline Creatinine mean (SD) $\mu \mathrm{mol} / \mathrm{l}$ & $\#$ & $218.34(192.7)$ & - & $\#$ & - \\
\hline Ureea mean (SD) mmol/l & $\#$ & $16.2(12.1)$ & - & $\#$ & - \\
\hline lactate (septic shock patients) mmol/l (SD) & $\#$ & $3.93(2.53)$ & - & $3.80(3.03)$ & 0.30 \\
\hline oliguria/anuria no of patients (\%) & $\#$ & $40(56.3)$ & - & $28(39.43)$ & 0.04 \\
\hline Capilary refill time $>3 \mathrm{sec}$ no of patients (\%) & $\#$ & $17(23.94))$ & - & $12(16.9)$ & 0.29 \\
\hline
\end{tabular}


The mortality rates at twenty-eight days among the two groups were not significantly different.

The all-cause mortality for all patients included in the study was similar to the one found in other reported studies on the subject $[2,23]$.

The findings of this study cannot be extrapolated due to the small number of included patients. Due to the limited research in the field of fluid resuscitation of the obese patients with sepsis and septic shock, it is difficult to say whether using ideal or adjusted weight for calculating the amount of fluid may have different outcomes in terms of mortality [18].

\section{CONCLUSION}

Advanced haemodynamic monitoring favoured adjusting the initial fluid load to the IBW. A lower than $30 \mathrm{ml} / \mathrm{kg}$ IBW initial fluid load was not found to be an aggravator factor for the persistence of sepsis-induced AKI.

\section{CONFLICT OF INTEREST}

None to declare.

\section{REFERENCES}

1. Sakhuja A, Kumar G, Gupta S, Mittal T, Taneja A, Nanchal RS. Acute Kidney Injury Requiring Dialysis in Severe Sepsis. Am J Respir Crit Care Med. 2015;192:951-7.

2. Angus DC, van der Poll T. Severe sepsis and septic shock. $N$ Engl J Med. 2013; 369:840-51.

3. Brown RM, Semler MW. Fluid Management in Sepsis. J Intensive Care Med. 2019;34:364-73.

4. Ince C. Personalized physiological medicine. Crit Care. 2017;21(Suppl 3):308.

5. Saugel B, Michard F, Scheeren TWL. Goal-directed therapy: hit early and personalize!. J Clin Monit Comput. 2018;32:375-77.

6. Dellinger RP, Levy MM, Rhodes A, et al. Surviving Sepsis Campaign Guidelines Committee including The Pediatric Subgroup. Surviving Sepsis Campaign: international guidelines for management of severe sepsis and septic shock, 2012. Intensive Care Med. 2013;39:165-228.

7. Rhodes A, Evans LE, Alhazzani W, et al. The Third International Consensus Definitions for Sepsis and Septic Shock (Sepsis-3). JAMA. 2016;315:801-10

8. Iwakiri Y, Shah V, Rockey DC. Vascular pathobiology in chronic liver disease and cirrhosis - Current status and future directions. J Hepatol. 2014;61:912-24.

9. Varin R, Mulder P, Tamion F, et al. Improvement of endothelial function by chronic angiotensin-converting enzyme inhibition in heart failure: role of nitric oxide, prostanoids, oxidant stress, and bradykinin. Circulation. 2000;102:351-6.

10. Laurent S, Cockcroft J, Van Bortel L, et al. European Network for Non-invasive Investigation of Large Arteries. Expert consensus document on arterial stiffness: methodological issues and clinical applications. Eur Heart J 2006;27:2588-605.

11. Mitchell GF, Parise H, Benjamin EJ,. Changes in arterial stiffness and wave reflection with advancing age in healthy men and women: the Framingham Heart Study. Hypertension. 2004;43:1239-45.

12. Levy MM, Evans LE, Rhodes A. The Surviving Sepsis Campaign Bundle: 2018 Update. Crit Care Med. 2018;46:997-1000.

13. Vincent JL, Moreno R, Takala J, Willatts S. The SOFA (Sepsisrelated Organ Failure Assessment) score to describe organ dysfunction/failure. On behalf of the Working Group on Sepsis-Related Problems of the European Society of Intensive Care Medicine. Intensive Care Med. 1996;22:707-10.

14. Knaus WA, Draper EA, Wagner DP, Zimmerman JE. APACHE II: A severity of disease classification system. Crit Care Med. 1985;13:818-29.

15. Khwaja A. KDIGO clinical practice guidelines for acute kidney injury. Nephron Clin Pract. 2012;120:179-84.

16. Mehta RL, Kellum JA, Shah SV, et al. Acute Kidney Injury Network. Acute Kidney Injury Network: report of an initiative to improve outcomes in acute kidney injury. Crit Care. 2007;11:R31.

17. Lemmens HJ, Brodsky JB, Bernstein DP. Estimating ideal body weight--a new formula. Obes Surg. 2005;15:1082-3.

18. Taylor SP, Karvetski CH, Templin MA, Heffner AC, Taylor BT. Initial fluid resuscitation following adjusted body weight dosing is associated with improved mortality in obese patients with suspected septic shock. J Crit Care. 2018 ;43:7-12.

19. Wacharasint P, Boyd JH, Russell JA, Walley KR. One size does not fit all in severe infection: obesity alters outcome, susceptibility, treatment, and inflammatory response. Crit Care. 2013;17:R122.

20. Arabi YM, Dara SI, Tamim HM, et al. Cooperative Antimicrobial Therapy of Septic Shock (CATSS) Database Research Group. Clinical characteristics, sepsis interventions and outcomes in the obese patients with septic shock: an international multicenter cohort study. Crit Care. 2013;17:R72.

21. Hjortrup PB, Haase N, Bundgaard H, et al. CLASSIC Trial Group; Scandinavian Critical Care Trials Group. Restricting volumes of resuscitation fluid in septic shock after the initial management: The CLASSIC randomised, parallel-group, multicenter feasibility trial. Intensive Care Med. 2016;42:1695-705.

22. Maitland K, George EC, Evans JA, et al. FEAST trial group. Exploring mechanisms of excess mortality with early fluid resuscitation: Insights from the FEAST trial. BMC Medicine. 2013;11:68

23. Fleischmann C, Thomas-Rueddel DO, Hartmann M,et al. Hospital Incidence and Mortality Rates of Sepsis. An Analysis of Hospital Episode (DRG) Statistics in Germany from 2007 to 2013. Dtsch Arztebl Int. 2016;113:159-66. 